\title{
Simmitecan Hydrochloride
}

National Cancer Institute

\section{Source}

National Cancer Institute. Simmitecan Hydrochloride. NCI Thesaurus. Code C106369.

The hydrochloride salt form of simmitecan, an ester prodrug of chimmitecan, a 9-alkyl substituted camptothecin derivative with potential antineoplastc activity. Upon intravenous administration, simmitecan is hydrolyzed by carboxylesterase and the activated form, chimmitecan, is produced. Chimmitecan inhibits topoisomerase I, stabilizes covalent topoisomerase I-DNA complexes, and inhibits the religation of topoisomerase I-mediated single-strand DNA breaks. Futhermore, the covalent topoisomerase I-DNA complexes interfere and block the DNA replication machinery, resulting in the production of potentially lethal double-strand DNA breaks. This leads to an inhibition of DNA replication and the induction of apoptosis. The modification at position 9 yields improved cytotoxicity compared to some other camptothecin analogues. 